\title{
The effects of pulse pressure on left atrium and left ventricle geometry in hypertensive patients
}

\author{
Arwa M. Fuzi Alsaraf \\ Department of Medicine, College of Medicine, University of Mosul.
}

(Ann. Coll. Med. Mosul 2010; 36 (1 \& 2): 56-62).

Received: $13^{\text {th }}$ Oct 2010; Accepted: $9^{\text {th }}$ Feb 2011.

\begin{abstract}
Objectives: An increased pulse pressure (PP) suggests aortic stiffening. New evidence suggests that $(\mathrm{PP})$ is more sensitive measure of cardiac risk than other indexes of blood pressure.

We aim to study the effect of pulse pressure in hypertensive patients on left atrial and left ventricle diameters (LAD, LVD), left ventricle geometry [wall thickness (WT), left ventricle mass (LVM), regional wall thickness (RWT)] and the effects of age and sex on the PP.
\end{abstract}

Methods: Echocardiography study of LAD, LVD, left ventricle end diastolic diameter (LVEDD), aortic root diameter (ARD), ratios and left ventricle geometry was performed on 92 patients (61 female \& 31 male). A correlation analysis between these variables and pulse pressure was done. Patients were grouped into high PP >60 mmHg and normal PP $<59 \mathrm{mmHg}$ and chi-square test was applied.

Results: We found strong positive correlation between age and PP ( $p$ value $=0.00$ ), positive correlation of PP with WT, LVM and SBP ( $p$ value < 0.05 ) and positive correlation of LVM to LAD, LVD, RWT, LAD/ARD, LAD/LVEDD and the age ( $p$ value <0.05).

Conclusion: The PP which increased by age is the most important BP measurement (compared to SBP and DBP) that directly affects LV geometry mainly WT \& LVM.

Keywords: Pulse pressure, left atrium, left ventricle, geometry, LV mass, wall thickness, regional wall thickness.

الأهداف: ان ضغط النبض المتز ايد يدل على وجود تصلب أبهري. الدلائل و البحوث الجديدة تشير الى أن قياس ضغط

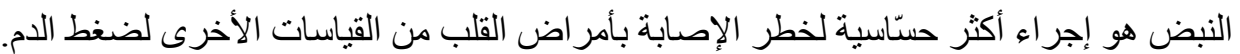

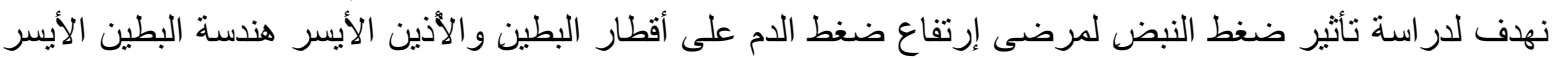

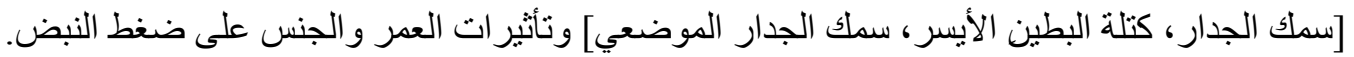

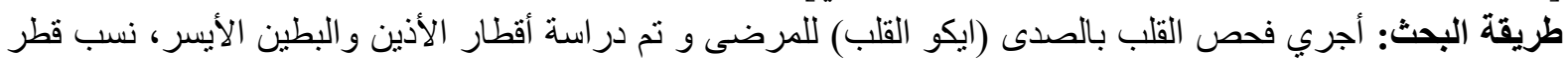

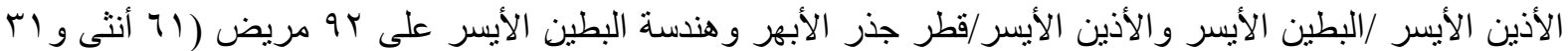

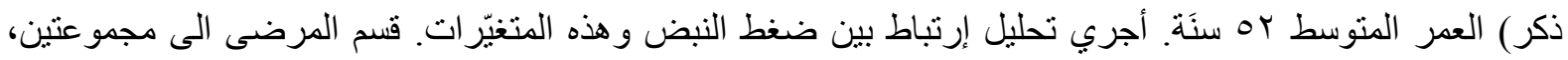

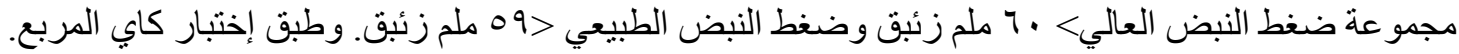

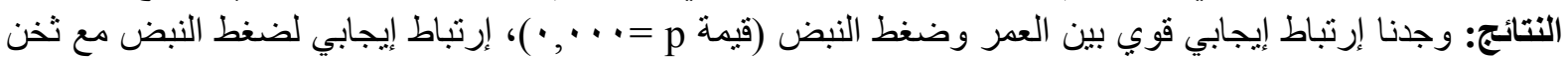
الجدار وكتلة البطين الأيسر (قيمة p> > •, • ) وإرتباط إيجابي لكتلة البطين الأيسر مع قطر الأذين والبطين الأيسر، سمك

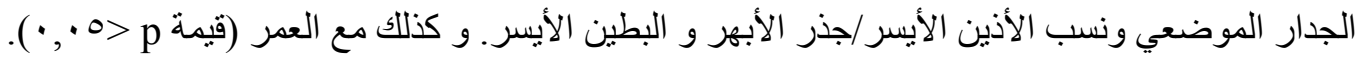

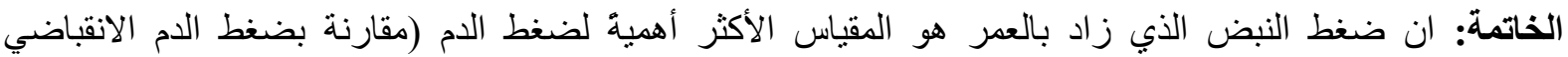
و الانبساطي)، لتأثيره المباشر على هندسة البطين الأيسر و بشكل رئيسي على سمك الجدار وكتلة البطين الأيسر. 
C urrent guidelines for the diagnosis and management of hypertension have defined cardiovascular risk by the elevation of systolic blood pressure (SBP) and/or the elevation of diastolic blood pressure (DBP) ${ }^{(1,2)}$. Recently research has focused on the relation between cardiovascular diseases and elevated pulse pressure (PP) which apparently reflects increased large artery stiffness ${ }^{(3,4)}$. The principal components of blood pressure (BP) consist of both a steady component (mean arterial blood pressure MAP) and a pulsatile component (pulse pressure PP). Major determinants of MAP are ventricular ejection and peripheral vascular resistance ${ }^{(1,5)}$. PP, the difference between SBP and DBP, is also made up of two major components one due to ventricular ejection and the elastic properties of the large arteries (direct) and the other due to wave reflection (indirect). The rise in SBP and DBP in middle aged and elderly subjects is due primarily to an increase in large artery stiffness and an associated increase in wave reflection amplitude ${ }^{(1)}$.

In older people, observational studies have commonly found $U$ or $S$ shaped relationship of BP with mortality especially for DBP; in some studies, individuals with the lowest BP had the highest mortality. It is possible that the PP is the measure of $\mathrm{BP}$ most strongly related to cardiovascular risk in elderly people. Several prospective studies have found that elevated PP is associated with risk of myocardial infarction, congestive heart failure and cardiovascular total mortality. It also increases the risk of stroke as a consequence of arterial stiffness and left atrial dilatation and subsequent risk of atrial fibrillation ${ }^{(6-8)}$.

As shown in the study (pulse pressure and mortality in older people 2000), PP appears to be the best single measure of $\mathrm{BP}$ in predicting mortality in older people ${ }^{(6)}$.

The exponential rise in atrial fibrillation incidence with age parallels a rapid age related increase in aortic stiffness ${ }^{(9)}$. The concomitant increase in PP adds to pulsatile load on the heart ${ }^{(10)}$, thereby promoting ventricular hypertrophy ${ }^{(9,11,12)}$, impaired ventricular relaxation ${ }^{(13,14)}$ and increased left atrial (LA) size ${ }^{(11)}$. This all together may lead to fibrosis and electrical remodeling in the atrium $^{(9)}$, leading to atrial fibrillation. Consistent with this causal pathway, echo measures of abnormal left ventricle (LV) geometry and diastolic dysfunction are associated with increased risk of atrial fibrillation ${ }^{(15,16)}$.

Increased LAD/LVD ratio was observed in subjects with hypertension, diabetes and LV hypertrophy ${ }^{(17)}$. Increased ratio predicted worse exercise capacity. These data are consistent with the hypothesis that this ratio is a non invasive marker of LV diastolic pressure - volume relationship ${ }^{(17)}$.

It is shown that PP is an independent predictor of increased incidence of coronary heart disease and congestive heart failure ${ }^{(18)}$, accordingly, the PP may represent an easily measured and potentially modifiable risk factor for cardiovascular risks.

Our objective is to measure the pulse pressure in hypertensive patients with different ages and sex and to study its effect on left atrium diameter (LAD), left ventricle geometry \{including left ventricle diameter (LVD), left ventricle wall thickness (WT), left ventricle mass (LVM) and regional wall thickness (RWT)\}, the ratio between LAD and both aortic root and LV diameters.

\section{Patients and methods}

This is a patient based cross section correlation and case control study. It began in October 2009 and ended in May 2010. One hundred twenty hypertensive males and females aged between 30 and 78 year old were enrolled.

\section{Study sample and data collection}

From October 2009 until May 2010 we recruited 120 patients with history of hypertension or receiving anti-hypertensive medications for 3 years or more, visiting the echography clinic in Ibn Sina Teaching Hospital in Mosul for different medical and preoperative checking indications.

Thorough history was taken including history of hypertension or antihypertensive medication for the last 3 years.

After taking a written consent from the patients, recordings of SBP and DBP were taken in the supported right arm of lying 
subject, after 5 minutes of quiet rest, using a mercury column sphygmomanometer. Readings were recorded to the nearest even number. SBP was recorded at the first appearance of Korotkoff sounds and palpation was used to check auscultatory systolic readings. DBP was recorded at the disappearance (phase 5) of Korotkoff sounds. Baseline SBP and DBP each were the average of two separate measurements taken by the examining physician.

A standardized 2- dimensional guided $\mathrm{M}$ mode echocardiogram was performed at baseline examination. The following data were obtained: diastolic LAD, aortic root diameter (ARD), LV end diastolic diameter (LVEDD), septal thickness (ST), posterior wall thickness (PWT) and ejection fraction (EF).

A standard 12 leads ECG was taken.

The patients with EF less than $55 \%$ and those with evidence of acute coronary ischemia or previous myocardial infarction were excluded from the study; the remaining 92 patients were included.

The mean of ST and PWT for each patient was calculated, and considered as WT, normal $(<12.5 \mathrm{~mm})$. The LAD was measured, normal $(<38 \mathrm{~mm})$. The LVEDD also obtained, $(<43 \mathrm{~mm})$ for female and $(<45 \mathrm{~mm})$ for male was considered small LV. The following ratios were measured; LAD/ARD ratio, normal $(<1.2 \mathrm{~mm})$ and LAD/LVEDD ratio, normal (<.65). The LVM was obtained from the following formula ${ }^{(19)}$ :

$0.83 X\left\{(\mathrm{ST}+\mathrm{PWT}+\mathrm{LVID})^{3}{ }^{3}\right.$ LVID $^{3}{ }^{3}$. (Normal $<180$ in female, $<225$ in male)

The RWT (index of pressure overload in patients with LV hypertrophy with concentric remodeling) was obtained from the following formula ${ }^{(20)}$ : 2PWT/LVIDD. (Normal <0.5)

The patients were classified according to their PP into two groups: those with $\mathrm{PP}<59$ $\mathrm{mm} \mathrm{Hg}$ were considered as control group and those with $\mathrm{PP}>60 \mathrm{~mm} \mathrm{Hg}$ were considered as high PP group, and then we study the percentage of patients with abnormal values in both PP groups.

\section{Statistical analysis}

The correlation coefficient $(r)$ was used to measure the strength of the association between the PP, SBP and DBP with each of the following variables: age, LAD, LVD, WT, LAD/ARD, LAD/LVEDD ratios, LVM and RWT, Then the SBP, DBP and PP were correlated to each others. $P$ value of $<0.05$ ( 2 - tailed) was considered significant.

Chi - square test was used to study the effect of sex on PP, and to compare the number of patients with normal and abnormal values in both PP groups. $P$ value $<0.05$ was considered significant.

\section{Results}

A total of 92 hypertensive patients for more than 3 years with a normal EF and no evidence of acute coronary ischemia or previous myocardial infarction were included in the study. Age was between 30-74 years, with a mean of 52 year (10.96 SD). There were 61 female patients $(66.3 \%)$ and 31 male patients (33.7\%).We found that $62 \%$ of females had high PP compared to $55 \%$ of males. ( $P$ value $=$ 0.491), no significant effect of sex on the PP.

Twenty four patients (44\%) with high PP were above 60 year, compared to 5 pt (13\%) with low PP were above 60 year. $(P$ value $=0.002)$, with significant positive correlation between age and PP (table 2).

Thirty six patients (65\%) with high PP had WT $>12.5$ compared to 19 pt (51\%) with low PP group (table 1), There was higher percentage of patients of high PP group with increased WT, $(p$ value $=0.17)$. By the correlation analysis there is positive correlation between PP\& WT ( $p$ value $=0.024)$ (table 2$)$.

The effect of PP on LVM was found to be highly significant, 43 patients (78\%) of high PP group had increased LVM, compared to 15 patients $(40 \%)$ of low PP group ( $p$ value $<0.05$ ) (table 1). This effect of PP on LVM was also proved by the correlation analysis (table 2), which found significant positive correlation between PP\& LVM, ( $p$ value $<0.05$ ).

There was statistically significant positive correlation of LVM with LAD and LVD ( $p$ value $=0.000$ ) for both, also it shows positive correlation with LAD/ARD and LAD/LVIDD ratios ( $p$ value $=0.006,0.03$ respectively). Direct significant positive correlation between age and LVM was also noticed ( $p$ value= 0.007) (table 5). 
Table (1): Comparison of normal and abnormal values of both PP groups.

\begin{tabular}{|c|c|c|c|c|}
\hline \multicolumn{2}{|c|}{ Pulse pressure } & $\begin{array}{c}<59 \mathrm{mmHg} \\
(\%)\end{array}$ & $\begin{array}{c}>60 \mathrm{mmHg} \\
(\%)\end{array}$ & $p$-value \\
\hline \multirow{2}{*}{ WT } & increased & $19(51 \%)$ & $36(65 \%)$ & \multirow{2}{*}{0.176} \\
\hline & normal & $18(49 \%)$ & 19(35\%) & \\
\hline \multirow{2}{*}{ RWT } & increased & $21(56 \%)$ & 38 (69\%) & \multirow{2}{*}{0.226} \\
\hline & normal & $16(44 \%)$ & 17 (31\%) & \\
\hline \multirow{2}{*}{ LVM } & increased & $15(40 \%)$ & $43(78 \%)$ & \multirow{2}{*}{$0.00^{*}$} \\
\hline & normal & $22(60 \%)$ & $12(22 \%)$ & \\
\hline \multirow{2}{*}{ LAD } & increased & $9(24 \%)$ & $36(65 \%)$ & \multirow{2}{*}{0.26} \\
\hline & normal & $28(76 \%)$ & $19(35 \%)$ & \\
\hline \multirow{2}{*}{ LVEDD } & small & $23(62 \%)$ & $38(69 \%)$ & \multirow{2}{*}{0.49} \\
\hline & large & $14(38 \%)$ & $17(31 \%)$ & \\
\hline \multirow{2}{*}{ LAD//ARD } & increased & $14(38 \%)$ & $31(56 \%)$ & \multirow{2}{*}{0.08} \\
\hline & normal & $23(62 \%)$ & $24(44 \%)$ & \\
\hline \multirow{2}{*}{ LAD/LVEDD } & increased & $17(46 \%)$ & 32 (58\%) & \multirow{2}{*}{0.24} \\
\hline & normal & $20(54 \%)$ & $23(42 \%)$ & \\
\hline
\end{tabular}

Chi- square test used

*p-value considered significant

Table (2): Correlation analysis between pulse pressure and other variables.

\begin{tabular}{|l|c|c|c|c|}
\hline $\begin{array}{c}\text { Pulse } \\
\text { pressure } \\
\text { correlated } \\
\text { with }\end{array}$ & Mean & Std & $(r)$ & $\begin{array}{c}\text { P value } \\
\text { Sig }(2- \\
\text { tailed) }\end{array}$ \\
\hline Age & 52.3 & 10.96 & 0.390 & $0.00^{\star}$ \\
\hline LAD & 29.39 & 3.89 & 0.169 & 0.106 \\
\hline LVEDD & 44.29 & 3.79 & 0.39 & 0.714 \\
\hline WT & 12.94 & 1.69 & 0.236 & $0.024^{*}$ \\
\hline LAD/ARD & 1.193 & 0.19 & 0.016 & 0.881 \\
\hline LA/LVEDD & 0.662 & 0.091 & 0.252 & 0.121 \\
\hline LVM & 216.92 & 50.2 & 0.249 & $0.017^{*}$ \\
\hline RWT & 0.582 & 0.09 & 0.185 & 0.078 \\
\hline
\end{tabular}

$(r)=$ correlation coefficient

* = significant at 0.05

From the high PP group 36 pt (65\%) had enlarged LA. Compared to 9 pt (24\%) from low PP group, $(p$ value $=0.29)$ (table 1). Although there was higher percentage of pt with high PP have enlarged LA, this was statistically insignificant. Furthermore the correlation analysis showed no significant correlation between PP \& LAD. P value $=0.106$ (table 2 ). Thirty eight patients (69\%) of high PP had small ventricles, compared to 23 (62\%) of low PP $(P$ value $=0.491)($ table 1$)$

The LAD/ARD ratio appears to be higher in high PP group but it is statistically insignificant, ( $p$ value $=0.08)$ (table 1$)$. Correlation study also shows no significant correlation, ( $p$ value $=0.88$ ) (table 2).

The LAD/LVEDD ratio was studied. Of high PP group 32pt (58\%) had high ratios, compared to 17 pt (46\%) of low PP group. (P value $=0.24)$, (table1). No significant correlation was found between PP and this ratio by correlation analysis ( $p$ value $=0.25$ ) (table 2).

No significant correlation was found between PP \& RWT, ( $p$ value> 0.05) (table 1, table 2).

The correlation analysis between the PP and each of the above studied variables is shown in (table 2). There is a positive correlation between PP and age, WT, LVM in total and LVM in female. There is no correlation between $P P$ and $L A D$, LAD/ARD ratio, LAD/LVEDD ratio, and PP with RWT.

There was a significant positive correlation between SBP and RWT, ( $p$ value= 0.0116). No significant correlation was found between SBP and the other parameters. 
There was significant negative correlation between DBP and age ( $p$ value $=0.004$ ) Although there was negative correlation of $P P$ with WT, LAD/ARD, LAD/LVEDD, LVM, LA, LV and $R F$, but it was statistically not significant ( $p$ value $>0.05$ ), also there was insignificant positive correlation with RWT, ( $p$ value 0.156 ) (table 4).

The SBP was positively correlated with both DBP and PP, ( $p$ value< 0.05 ) (table 3 ).

Table (3): Correlation analysis between SBP and other variables

\begin{tabular}{|l|c|c|}
\hline SBP correlated with & $(\mathrm{r})$ & P value \\
\hline Age & 0.185 & 0.077 \\
\hline LAD & 0.126 & 0.231 \\
\hline LVEDD & 0.011 & 0.917 \\
\hline WT & -0.172 & 0.101 \\
\hline LAD/ARD & -0.061 & 0.563 \\
\hline LAD/LVEDD & 0.086 & 0.415 \\
\hline LVM & 0.144 & 0.170 \\
\hline RWT & 0.262 & $0.011^{*}$ \\
\hline PP & $0.828^{\star}$ & $0.00^{*}$ \\
\hline DBP & $0.532^{*}$ & $0.00^{*}$ \\
\hline
\end{tabular}

$(r)=$ correlation coefficient

* = significant at 0.05

Table (4): Correlation analysis between DBP and other variables.

\begin{tabular}{|l|c|c|}
\hline DBP correlated with & $(r)$ & P value \\
\hline Age & $-0.297^{*}$ & $0.004^{*}$ \\
\hline LAD & -0.098 & 0.352 \\
\hline LVEDD & -0.031 & 0.769 \\
\hline WT & -0.037 & 0.726 \\
\hline LAD/ARD & -0.131 & 0.213 \\
\hline LAD/LVEDD & -0.061 & 0.56 \\
\hline LVM & -0.036 & 0.73 \\
\hline RWT & 0.149 & 0.156 \\
\hline PP & -0.021 & 0.84 \\
\hline
\end{tabular}

$(r)=$ correlation coefficient

* = significant at 0.05
Table (5): Correlation analysis between LVM and other variables.

\begin{tabular}{|l|c|c|}
\hline LVM correlated with & $(r)$ & P value \\
\hline LAD & 0.478 & $0.00^{*}$ \\
\hline LVEDD & 0.405 & $0.00^{*}$ \\
\hline WT & 0.082 & 0.43 \\
\hline LAD/ARD & 0.282 & $0.006^{*}$ \\
\hline LAD/LVEDD & 0.218 & $0.03^{*}$ \\
\hline RWT & 0.340 & $0.00^{*}$ \\
\hline Age & 0.275 & $0.007^{*}$ \\
\hline
\end{tabular}

$(r)=$ correlation coefficient.

\section{Discussion}

This study shows significant positive correlation between age and $\mathrm{PP},(p$ value $=$ 0.000 ), and negative correlation between DBP and age $(p$ value $=0.004)$. Although the SBP has positive correlation with age, but it is statistically insignificant ( $p$ value $=0.07$ ).

As large artery stiffness increases in middle aged and elderly, SBP rises and DBP falls, with a resulting increase in $\mathrm{PP}^{(26)}$. The normally present higher gradient of peripheral to central arterial PP (amplification) found in young subjects gradually decreases with aging as a result of the augmentation of central PP by early wave reflection ${ }^{(27)}$.

As shown in table $3 \& 4$ the SBP was significantly positively correlated with both DBP\&PP ( $p$ value $=0.000)$. DBP was negatively correlated with $\mathrm{PP}$, but it was an insignificant correlation ( $p$ value $=0.84$ ). So in our study the $\mathrm{PP}$ is mainly determined by the high SBP

As vascular resistance rises, there is proportional increase in SBP and DBP in young individuals. With the onset of middle age, however, SBP rises more than DBP, resulting in elevation of $\mathrm{PP}^{(26,28)}$. Thus, DBP rises with increased peripheral arterial resistance and falls with increased central artery stiffness; the relative contributions of these 2 opposing forces determine DBP and ultimately PP. Normally, PP \& SBP are highly correlated because both BP components rise with increases in vascular resistance and large artery stiffness ${ }^{(1)}$. Determinants of PP include
* = significant at 0.05 rather than low DBP. 
ventricular ejection and stroke volume, but the age dependent increase in PP is largely determined by the increase in stiffness in large $\operatorname{arteries}^{(21)}$. Recently, it was shown that telomeres, the ends of chromosomes, which serve as clocks of cellular aging, are inversely related to $\mathrm{PP}^{(21,22,23)}$. Therefore, pp might serve as a phenotype of chronological and biological aging $^{(21)}$.

The WT is positively correlated with PP ( $p$ value $<0.05$ ) (table 1 , table 2 ). There is also significant positive correlation between LVM and PP, ( $p$ value<0.05), (table 1 , table 2$)$. Although there is positive correlation between $P P$ \& LAD, LAD/ARD, LAD/ LVIDD, LAD/LVMD, and negative correlation between PP \&LVD, this was statistically insignificant ( $p$ values $>0.05$ ). So in our study the main echo parameters affected by PP is the WT\& LVM.

The LVM has significant positive correlation with RWT, LAD/LVIDD, LAD, LVD and also with age, ( $p$ values $<0.05$ ), (table 5 ). So the LVM is a very important single echo parameter affecting other echography risks for heart diseases.

In previous studies it was found that in middle aged and elderly coronary heart disease risk increased with higher $\mathrm{PP}^{(10)}$, neither SBP nor DBP was superior to PP in predicting coronary heart disease risk ${ }^{(1)}$.

Elevated PP predisposes to myocardial infarction, heart failure ${ }^{(9,24)}$, left ventricle hypertrophy ${ }^{(11)}$, impaired ventricle relaxation ${ }^{(11,14)}$, increased left atrial size and atrial fibrillation ${ }^{(8)}$. The (pulse pressure and risk of new onset atrial fibrillation 2007) study ${ }^{(9)}$, showed that PP is the single BP component most predictive of future development of atrial fibrillation. In these multivariable models, the association between PP and atrial fibrillation risk persist in models that included echocardiographic LVM, LA size and time dependent change in elecetrocardiographic LV hypertrophy ${ }^{(9)}$.

The PP appears to be the best single measure of BP in predicting mortality in older people and helps to explain apparently discrepant results for low $\mathrm{DBP}^{(25)}$. Consideration of PP may explain the apparent increased risk associated with low DBP ${ }^{(25)}$.
There is significant correlation between SBP and RWT ( $p$ value $=0.011$ ), (table 3 ). This is explained because the RWT is most sensitive useful index of evaluation of patient with LV hypertrophy, concentric re-modeling even if the LVM is normal, ${ }^{(20)}$. So it is easily affected by SBP elevation before PP starting to increase. The DBP appears to be negatively correlated with WT. LVM. LAD/LAD \& LVIDD ratios, RWT, LAD and LVD (table 4), but it was statistically insignificant ( $p$ value $>0.05$ ). So the DBP has no effect on echography risks for heart diseases.

\section{Conclusion}

In conclusion our study shows that PP which reflects increased arterial stiffness in middle aged and elderly hypertensive patients is strongly correlated with age and it is the main BP component that affects the LV geometry specially LVM \& WT, which explain the increased cardiovascular risks and mortality in elderly patients. So our data suggests that changes in LV geometry are more related to the pulsatile stress of large artery stiffness during systole as reflected by rise of $\mathrm{PP}$, than the steady - state stress of resistance during diastole as reflected by parallel rise in SBP\& DBP.

\section{Recommendation}

Further studies are needed to determine the relative values of antihypertensive therapies and whether reduction of PP should be specific treatment target to reduce cardiovascular risks and mortality in middle aged and elderly hypertensive patients.

\section{Referrences}

1. Stanley SF, Shehzad AK, Nathan DW, Mortin GL, Danial L. is pulse pressure useful in predicting risk for coronary heart disease?. The Framingham Heart Study. American Heart Association, 1999.

2. Joint National Committee on Prevention, Detection, Evaluation and Treatment of High Blood Pressure, The sixth report. Arch Intern Med. 1997; 157:2413-2446.

3. Michael D, Gary M, Mare P, et al. pulse pressure and cardiovascular disease related mortality. JAMA, 2002; 287:26772683. 
4. Psaty BM, Furberg CD, Kuller LH, et al. Association between blood pressure level and the risk of myocardial infarction, stroke and total mortality. Arch Intern Med. 2001; 161:1183-1192.

5. Nicholas WW, O' Rourk MF, McDonald' s. blood flow in arteries. Philadelphia, $\mathrm{Pa}$ : Lea and Fibiger; 1998.

6. Robert J, Glynn, et al, pulse pressure and mortality in older people, Arch Intern Med. 2000; 160: 2765-2772.

7. Franklin SS, Khan SA, Wong ND, Larson $M G$, Levy $D$. is pulse pressure useful in predicting risk for coronary heart disease? The Framingham Heart Study. American Heart Association, Circulation. 1999; 100: 354-360.

8. Lanteleme $\mathrm{P}$, Laurent $\mathrm{S}$, Besnard $\mathrm{C}$, et al. Arterial stiffness is associated with left atrial size in hypertensive patients. Arch Cardiovasc Dis. 2008; 101:9-10.

9. Gary F, Mitchell MD, et al. pulse pressure and risk of new onset atrial fibrillation, JAMA. 2007; 297: 709.

10. Mitchell GF, Parise H, Benjamin EJ, et al. Changes in arterial stiffness and wave reflection with advancing age in healthy men \& women. The Framingham Heart Study. Hypertension. 2004; 43: 12391245.

11. Tigan $\mathrm{K}$, Karaahet $\mathrm{T}$, Fotbolen $\mathrm{H}$, et al. The influence of dipper and non dipper blood pressure patterns on left ventricular functions in hypertensive patients: a tissue Doppler study. Turk Kardiyol Dern Ars. 2009; 37:101-106.

12. Libhaber E, Woodiwiss AJ, Libhaber C, et al. Gender specific brachial artery blood pressure independent relationship between pulse wave velocity and left ventricle mass index in a group of African ancestry. J Hypertens, 2008; 26:16191628.

13. Aqoston CL, Mocan T, Bober C. arterial stiffness and left ventricle diastolic function in the patients with arterial hypertension. Rom J Intern Med. 2008; 46: 313-321.

14. Hundiy WG, Kitzman DW, Morgan TM, et al. Cardiac cycle dependent changes in aortic area and distensibility are reduced in older patients with exercise intolarence. J Am Coll Cardiol. 2001; 38:796-802.

15. Tsang TS, Gersh RT, Appletion CP, et al. Left ventricular diastolic dysfunction as a predictor of the first diagnosed non valvuler atrial fibrillation in 840 elderly men and women. J Am Coll Cardiol. 2002; 40: 1636- 1644.
16. Vasan RS, Larson MG, Levy D, Galders $M$, Wolf PA, Benjamin EJ. Doppler transmitral flow indexes and risk of atrial fibrillation, the Framingham Heart Study. Am J Cardiol. 2003; 91: 1079- 1083.

17. Sperack DM, Blum L, Malhotra D, et al. Ratio of left atrial to left ventricle size: an anatomical marker of the diastolic left ventricular pressure - volume relationship. Echography 2008; 25:366-373.

18. Viola V, Thedore RH, Harkin MK. Pulse pressure and risk for myocardial infarction and heart failure in the elderly. J Am Coll Cardiol. 2000; 36:130-138.

19. Echocardiographic Evaluation of Left and Right ventricular Systolic Function. In Text Book of Clinical Echocardiology, editor Catherine MO: USA, 2000; P 104.

20. Echocardiography: a Practical Guide for Reporting, Editor Helen R, Jhon PC, 2007 inform UK Itd, P 30-31.

21. Nawrot TS, Steassen JA, Thijis L, et al. Should pulse pressure become part of the Framingham Risk Score? Journal of Human Hypertension 2004; 18: 279-286

22. Aviv A, Aviv H. Telomeres and essential hypertension. 1999; 12:427-432

23. Benetos $A$, Telemer length as an indicator of biological aging: the gender effect and relation with pulse pressure and pulse wave velocity. Hypertension. 2001; 37: 381-385.

24. Chea CU, Pfereffer MA, Glynn RJ, Mitchell GF, et al. increased pulse pressure and risk of the heart failure in the elderly. JAMA. 1999; 281:634-639.

25. Robert J, Glynn CU, Chae JM, et al. Pulse pressure and mortality in older people. Arch Intern Med. 2000; 160:2765- 2772.

26. Berne RM, Levy MN. Cardiovascular physiology. St Louis, Mo: CV Mosby 1992; 113-144.

27. Benetos S, Laurent $S$, Hoeks AD, Boutouyrie $\mathrm{PH}$, Safar ME. Arterial alterations with aging and high blood pressure. Arterioscler Thromb. 1993; 13: 90-97.

28. Franklin SS, Gustin WG, Wong ND, Lerson MG, et al. Hemodynamic patternes of age related changes in blood pressure: the Framingham Heart Study. Circulation 1997; 96:308-315. 